\title{
Article
}

\section{Dyslipidemia Management in Patients with Coronary Artery Disease. Data from the POLASPIRE Survey}

\author{
Piotr Jankowski ${ }^{1,2, *} \mathbb{D}$, Paweł Kozieł ${ }^{2}$, Małgorzata Setny ${ }^{3} \mathbb{( D}$, Marlena Paniczko ${ }^{4}\left(\mathbb{D}\right.$, Maciej Haberka ${ }^{5}$, \\ Maciej Banach 6 ${ }^{(D}$, Dirk De Bacquer ${ }^{7}$, Guy De Backer ${ }^{7}{ }^{\circ}$, Kornelia Kotseva ${ }^{8,9}$, David Wood ${ }^{9,10}$, \\ Zbigniew Gąsior ${ }^{5}$, Karol Kamiński ${ }^{4}$, Dariusz A. Kosior ${ }^{3,11}$ and Andrzej Pająk ${ }^{12(1)}$
}

1 Department of Epidemiology and Health Promotion, School of Public Health, Medical Centre of Postgraduate Education, 01-813 Warsaw, Poland

2 Institute of Cardiology, Jagiellonian University Medical College, 31-008 Kraków, Poland; tragez88@wp.p1

3 Department of Cardiology and Hypertension with the Electrophysiological Lab, Central Research Hospital the Ministry of The Interior and Administration, 00-124 Warsaw, Poland; malgorzata.setny@cskmswia.gov.pl (M.S.); dkosior13@gmail.com (D.A.K.)

4 Department of Population Medicine and Lifestyle Diseases Prevention, Medical University of Bialystok, 15-089 Bialystok, Poland; Marlena.paniczko@umb.edu.pl (M.P.); karol.kaminski@umb.edu.pl (K.K.)

5 Department of Cardiology, Medical University of Silesia, 40-055 Katowice, Poland; mhaberka@op.pl (M.H.); zgasior@sum.edu.pl (Z.G.)

6 Department of Hypertension, Chair of Nephrology and Hypertension, Medical University of Lodz, 93-338 Lodz, Poland; maciejbanach@aol.co.uk

7 Department of Public Health and Primary Care, Ghent University, 9000 Ghent, Belgium; dirk.debacquer@ugent.be (D.D.B.); guy.debacker@ugent.be (G.D.B.)

8 Imperial College Healthcare NHS Trust, London W2 1NY, UK; kornelia.kotseva@nuigalway.ie

check for

updates

Citation: Jankowski, P.; Kozieł, P.; Setny, M.; Paniczko, M.; Haberka, M.; Banach, M.; De Bacquer, D.;

De Backer, G.; Kotseva, K.; Wood, D.; et al. Dyslipidemia Management in Patients with Coronary Artery Disease. Data from the POLASPIRE Survey. J. Clin. Med. 2021, 10, 3711. https://doi.org/10.3390/jcm10163711

Academic Editor: Michalina Kołodziejczak

Received: 8 June 2021

Accepted: 17 August 2021

Published: 20 August 2021

Publisher's Note: MDPI stays neutral with regard to jurisdictional claims in published maps and institutional affiliations.

Copyright: (c) 2021 by the authors. Licensee MDPI, Basel, Switzerland. This article is an open access article distributed under the terms and conditions of the Creative Commons Attribution (CC BY) license (https:// creativecommons.org/licenses/by/ $4.0 /)$.
9 National Institute of Preventive Cardiology, National University of Ireland-Galway, H91 TK33 Galway, Ireland; d.wood2@imperial.ac.uk

10 Cardiovascular Medicine, National Heart and Lung Institute, Imperial College, London W2 1NY, UK

11 Faculty of Medicine, Medical College, Cardinal Stefan Wyszyński University, 01-938 Warsaw, Poland

12 Department of Clinical Epidemiology and Population Studies, Institute of Public Health, Jagiellonian University Medical College, 31-066 Kraków, Poland; andrzej.pajak@uj.edu.pl

* Correspondence: piotrjankowski@interia.pl; Tel.: +48-124-002-150; Fax: +48-124-002-167

Abstract: Lipid-lowering in patients with coronary artery disease (CAD) is related to a lower risk of cardiovascular events. We evaluated factors related to the management of hypercholesterolemia in patients with established CAD. Patients were interviewed 6-18 months after hospitalization for an acute coronary syndrome (ACS) or a myocardial revascularization procedure. Statins were prescribed at discharge to $94.4 \%$ of patients, while $68.1 \%$ of the patients hospitalized for an ACS were prescribed a high-dose statin. Hospitalization in a teaching hospital, percutaneous coronary intervention, cholesterol measurement during hospitalization and the male sex were related to prescription of statins at discharge. The intensity of lipid-lowering therapy in the post-discharge period increased in $17.3 \%$, decreased in $11.7 \%$, and did not change in $71.0 \%$ of the patients. The prescription of a lipid-lowering drug (LLD) at discharge (odds ratio 5.88 [95\% confidence intervals 3.05-11.34]) and a consultation with a cardiologist (2.48 [1.51-4.08]) were related to the use of LLDs, while age (1.32 [1.10-1.59] per 10 years), loneliness (0.42 [0.19-0.94]), professional activity (1.56 [1.13-2.16]), and diabetes (1.66 [1.27-2.16]) were related to achieving an LDL cholesterol goal 6-18 months after discharge. In conclusion, health-system-related factors are associated with the LLD utilization, whereas mainly patient-related factors are related to the control of hypercholesterolemia following hospitalization for CAD.

Keywords: cholesterol; coronary artery disease; hypercholesterolemia; secondary prevention

\section{Introduction}

Patients with established coronary artery disease (CAD) are at a high risk of recurrent cardiovascular events [1]. Despite advances in pharmacological and invasive 
treatment, risk factors remain independent predictors of cardiovascular mortality in CAD patients [2]. One of the most important of these is hypercholesterolemia, while the use of lipid-lowering drugs is related to an improved prognosis [3]. The European Society of Cardiology guidelines consider lowering LDL cholesterol as the cornerstone of cardiovascular prevention $[4,5]$. Despite overwhelming evidence for the benefits of lowering cholesterol levels, especially when using statins, a majority CAD patients still have LDL cholesterol levels above the recommended goal [6-9]. Moreover, although survivors of an acute coronary syndrome should be prescribed high-dose statins, most patients take lower doses [10].

Recent surveys indicated that there is considerable potential for further improvements in the secondary prevention of CAD in European countries [11-13]. Many intervention methods aimed at improving secondary prevention in CAD patients have been proposed previously [14-19]. Additionally, several factors influencing the quality of secondary prevention in every-day practice have been identified [20-22]. Nevertheless, identifying the remaining barriers to effective risk factor control is essential to ensure the maximum benefits of prevention interventions. The aim of the present analysis was to investigate the factors affecting the management of hypercholesterolemia in patients with established CAD.

\section{Materials and Methods}

The POLASPIRE study was a cross-sectional, multicenter survey designed to evaluate the implementation of the European guidelines for secondary prevention of CAD by assessing both the control of the main risk factors and the prescription rates of cardioprotective medication in patients with established CAD. The survey has been described in detail elsewhere [8]. In brief, fourteen departments of cardiology from twelve different hospitals participated. Seven of the departments were situated in teaching hospitals and seven in municipal hospitals. The inclusion criteria included hospitalization for an acute coronary syndrome (ACS) or a myocardial revascularization procedure and age from $\geq 18$ years to $\leq 80$ years. Data collection using standardized methods and the same instruments in all centers were utilized by centrally trained research staff. Overall, 1236 patients were invited to participate in the study and their medical records were reviewed.

The follow-up interviews were completed 6-18 months after discharge from the hospital. A patient's personal medical history, lifestyle and medications used were assessed using a standard questionnaire. The participants' education was assessed based on the number of years of formal education completed. Self-perceived income was based on the answers to the question: "In your opinion, your family income is: very low, low, middle, high". We looked at a measure of loneliness by including the question: "Do you have somebody with whom you share your problems or happiness?". We constructed a socio-economic status (SES) summary score based on the different socio-economic components [23]. This score was the sum of the following sub-scores: educational level (primary school completed or less $=0$, intermediate $=2$, college / university $=4$ ); perceived income (very low $=0$, low $=2$, intermediate $=4$, high $=6$ ); loneliness (yes $=0$, no $=2$ ); employment (yes $=2$, no $=0$ ); and being married (yes $=1$, no $=0$ ). Based on this summary score, varying from 0 to 15, we subdivided our sample in two groups: patients at a "low SES level" that had a summary score of $\leq 7$, and patients at a "high SES level" that had a score of $\geq 8$. The psychosocial characteristics of the patients were assessed on the basis of the Hospital Anxiety and Depression Scale [24].

Standard scales with a vertical ruler (SECA Medical Measuring Systems and Scales, Birmingham, UK) were used to complete measurements.

The scales were calibrated at the start of the survey. Height and weight were measured in a standing position without shoes and heavy outerwear. Body mass index (BMI) was calculated according to the following formula: $\mathrm{BMI}=$ weight $[\mathrm{kg}] /(\text { height }[\mathrm{m}])^{2}$. Obesity was defined as a BMI $\geq 30 \mathrm{~m} / \mathrm{kg}^{2}$. Smoking at the time of interview was defined as self-reported smoking verified by the concentration of breath carbon monoxide using a Smokerlyzer device (Model Micro+, Bedfont Scientific, Kent, UK). A high breath carbon 
monoxide was defined as $10 \mathrm{ppm}$ or more. Blood pressure was measured twice, on the right arm in a sitting position after at least five minutes of rest with an automatic digital Omron Comfort M6 sphygmomanometers (OMRON Corporation, Kyoto, Japan). High blood pressure was defined as a blood pressure $\geq 140 / 90 \mathrm{mmHg}$ or $\geq 140 / 85 \mathrm{mmHg}$ in diabetic patients [4]. A fasting venous blood sample was acquired to measure plasma lipid and creatinine levels. The results of analyses performed no later than $12 \mathrm{~h}$ after blood collection were used for the purposes of the present report. A high cholesterol level was defined as an LDL cholesterol $\geq 1.8 \mathrm{mmol} / \mathrm{L}$, whereas a high non-HDL cholesterol was defined as $\geq 2.6 \mathrm{mmol} / \mathrm{L}$ [4]. A high dose of statins was defined as atorvastatin in a dose of at least $40 \mathrm{mg}$ per day or rosuvastatin in a dose of at least $20 \mathrm{mg}$ per day. Highintensity cholesterol-lowering therapies were defined as a high-dose statin or any statin combined with the use of ezetimibe or a fibrate. All other lipid-lowering drug therapies were considered of "low or moderate intensity". The glomerular filtration rate was defined using the MDRD formula [25].

The survey's protocol was approved by the institutional Bioethics Committees. The study protocol complies with the ethical guidelines of the Declaration of Helsinki. All patients signed the informed consent form.

\section{Statistical Analysis}

Qualitative variables were reported as percentages and continuous variables as means (standard deviation, SD) or median (interquartile range, IQR). The Pearson $\chi 2$ or Fisher's exact test were used in the case of qualitative variables. The Shapiro-Wilk test was utilized to assess the normality of data. Normally distributed continuous variables were compared using the Student's t-test. Variables without normal distributions were assessed using the Mann-Whitney U-test. Multivariable, stepwise logistic regression analysis was performed to assess the factors independently related to the dependent variables. The initial models comprised all variables mentioned in Table 1. A two-tailed $p$-value of less than 0.05 was regarded as statistically significant. Statistical analyses were completed using the STATISTICA 13 software (TIBCO Software, Palo Alto, CA, USA).

\section{Results}

\subsection{In-Hospital Management}

The medical records of 1236 patients were reviewed and included in the analyses, of whom $354(29 \%)$ were females and $882(71 \%)$ were males. The characteristics of the study population are presented in Table 1.

Table 1. Characteristics of the study group.

\begin{tabular}{cc}
\hline Variable & Median or Number \\
\hline Age, years, median (IQR) & $65.3(59.9-71.3)$ \\
Sex & $882(71.4)$ \\
Males, $n(\%)$ & $354(28.6)$ \\
Females, $n(\%)$ & $481(38.9)$ \\
Recruiting event & $258(20.9)$ \\
Myocardial infarction, $n(\%)$ & $443(35.8)$ \\
Unstable angina, $n(\%)$ & $54(4.4)$ \\
PCI, $n(\%)$ & $903(73.1)$ \\
CABG, $n(\%)$ & $528(51.5)$ \\
Hospitalization in a teaching hospital, $n(\%)$ & $12.0(11.0-14.0)$ \\
Previous hospitalization for cardiovascular disease, $n(\%){ }^{a}$ & $750(73.2)$ \\
Duration of education, years, median $(\mathrm{IQR})$ a & $85(8.3)$ \\
Marital status a & $153(14.9)$ \\
Married, $n(\%)$ &
\end{tabular}


Table 1. Cont.

\begin{tabular}{|c|c|}
\hline Variable & Median or Number \\
\hline Never married, $n(\%)$ & $37(3.6)$ \\
\hline Living alone, $n(\%)^{a}$ & $161(15.7)$ \\
\hline Loneliness, $n(\%)^{\text {a }}$ & $37(3.1)$ \\
\hline \multicolumn{2}{|l|}{ Household income $^{\text {a }}$} \\
\hline High, $n(\%)$ & $48(4.7)$ \\
\hline Medium, $n(\%)$ & $655(63.9)$ \\
\hline Low, $n(\%)$ & $274(26.5)$ \\
\hline Very low, $n(\%)$ & $48(4.6)$ \\
\hline Employed, $n(\%)$ a & $316(30.8)$ \\
\hline High socio-economic status, $n(\%)^{a}$ & $387(37.8)$ \\
\hline Participation in a cardiac rehabilitation program following discharge, $n(\%)^{\text {a }}$ & $297(29.0)$ \\
\hline Regular physical activity & $140(13.7)$ \\
\hline \multicolumn{2}{|l|}{ Physician specialty ${ }^{a}$} \\
\hline Cardiologist, $n(\%)$ & $879(85.8)$ \\
\hline General practitioner, $n(\%)$ & $880(85.9)$ \\
\hline Diabetologist, $n(\%)$ & $111(10.8)$ \\
\hline Other physician, $n(\%)$ & $28(2.7)$ \\
\hline No physician, $n(\%)$ & $8(0.8)$ \\
\hline Smoking, $n(\%)^{\text {a }}$ & $176(17.2)$ \\
\hline Obesity, $n(\%)^{\text {a }}$ & $433(42.2)$ \\
\hline Diabetes, $n(\%)^{\mathrm{a}}$ & $408(39.8)$ \\
\hline High blood pressure, $n(\%)$ a,c & $433(42.2)$ \\
\hline High LDL cholesterol, $n(\%)$ a,d & $643(62.7)$ \\
\hline GFR $<60 \mathrm{~mL} / \mathrm{kg} / 1.73 \mathrm{~m}^{2}, n(\%)^{\mathrm{a}}$ & $185(18.0)$ \\
\hline Depression score, median (IQR) ${ }^{a, e}$ & $5.0(3.0-8.0)$ \\
\hline Anxiety score, median (IQR) & $6.0(3.0-8.0)$ \\
\hline
\end{tabular}

Abbreviations: CABG, coronary artery bypass grafting; GFR, glomerular filtration rate; IQR, interquartile range; LDL, low-density lipoprotein; PCI, percutaneous coronary intervention. a Among subjects who participated in the follow-up examination. ${ }^{\mathrm{b}}$ Hospitalization before the recruiting event due to: coronary artery bypass grafting; percutaneous coronary intervention; acute coronary syndrome; chronic coronary syndrome; heart failure; stroke; or peripheral artery disease. ${ }^{c}$ Blood pressure $\geq 140 / 90 \mathrm{mmHg}$ or $\geq 140 / 85 \mathrm{mmHg}$ in diabetics. ${ }^{\mathrm{d}}$ LDL cholesterol $\geq 1.8 \mathrm{mmol} / \mathrm{L}$. ${ }^{\text {e }}$ Based on the Hospital Anxiety and Depression Scale.

The patients' total cholesterol levels were available in 997 (80.7\%) hospital records, whereas LDL cholesterol measurements were found in 994 (80.4\%) records. The only factors significantly related to the availability of total cholesterol values in the medical records were age and the index event (Table 2). Among patients hospitalized for ACS, the total cholesterol measurement was found in $633(85.7 \%)$ hospital records, whereas LDL cholesterol measurements were available in $630(85.3 \%)$ hospital records. The total cholesterol availability in the hospital records of patients hospitalized for ACS was independently related to age, hospitalization in a teaching hospital and hospitalization for a myocardial infarction (Table 2).

A statin was prescribed at discharge to $1167(94.4 \%)$ patients with significant variations between departments ( $p<0.001$; Figure 1). A total of $839(67.9 \%)$ patients were prescribed a high-dose statin. The multivariable logistic analysis showed that hospitalization in a teaching hospital, percutaneous coronary intervention as an index event, cholesterol measurement during hospitalization and sex were independently related to the prescription of statins (Table 2). Among the patients prescribed a statin, 897 (77.1\%) were prescribed atorvastatin, $221(19.0 \%)$ were prescribed rosuvastatin, and $45(3.9 \%)$ were prescribed simvastatin. The mean dose in the case of atorvastatin was $46.2 \pm 27.2 \mathrm{mg}$ per day, in the case of rosuvastatin it was $19.5 \pm 11.1 \mathrm{mg}$ per day, and in the case of simvastatin it was $24.7 \pm 9.7 \mathrm{mg}$ per day. Hospitalization for myocardial infarction was the only factor independently related to the prescription of atorvastatin compared to the other statins (odds ratio 2.88 [95\% confidence intervals 2.09-3.99]). Among all studied patients, 41 (3.3\%) patients were prescribed a fibrate and $16(1.3 \%)$ were prescribed ezetimibe. No patient 
was prescribed any other lipid-lowering drug. Overall, 1171 (94.7\%) of all patients were prescribed at least one lipid-lowering drug.

Table 2. Factors independently related to the availability of a total cholesterol measurement during hospitalization and to the prescription of a statin at discharge from the hospital.

Variable Odds Ratio (95\% Confidence Intervals)

Factors Independently Related to the Availability of a Total Cholesterol Measurement During Hospitalization All patients $(n=1236)$

Age, per 10 years

Index event

Coronary artery bypass grafting

Percutaneous coronary intervention Unstable angina

Myocardial infarction

Patients hospitalized for an acute coronary syndrome $(n=739)$

Age, per 10 years

Hospitalization in a teaching hospital

Hospitalization for myocardial infarction
$0.82(0.68-0.99)$

$1.89(1.05-3.41)$

$2.46(1.31-4.61)$

$4.96(2.67-9.22)$

$0.72(0.56-0.94)$

$1.66(1.09-2.54)$

$1.91(1.26-2.92)$

Factors independently related to the prescription a statin at discharge from the hospital Dependent variable: statin in any dose; all patients $(n=1236)$

Hospitalization in a teaching hospital

Percutaneous coronary intervention as an index event

Total cholesterol measurement during hospitalization Males vs. females
$2.90(1.75-4.81)$

$2.52(1.32-4.84)$

$2.27(1.31-3.92)$

$2.00(1.21-3.31)$

Dependent variable: high-dose statin among patients hospitalized for an acute coronary syndrome $(n=739)$

Hospitalization for myocardial infarction

$3.82(2.71-5.38)$

Hospitalization in a teaching hospital

$2.41(1.70-3.40)$

$2.51(1.60-3.92)$

$1.49(1.04-2.14)$

Total cholesterol measurement during hospitalization Males vs. females

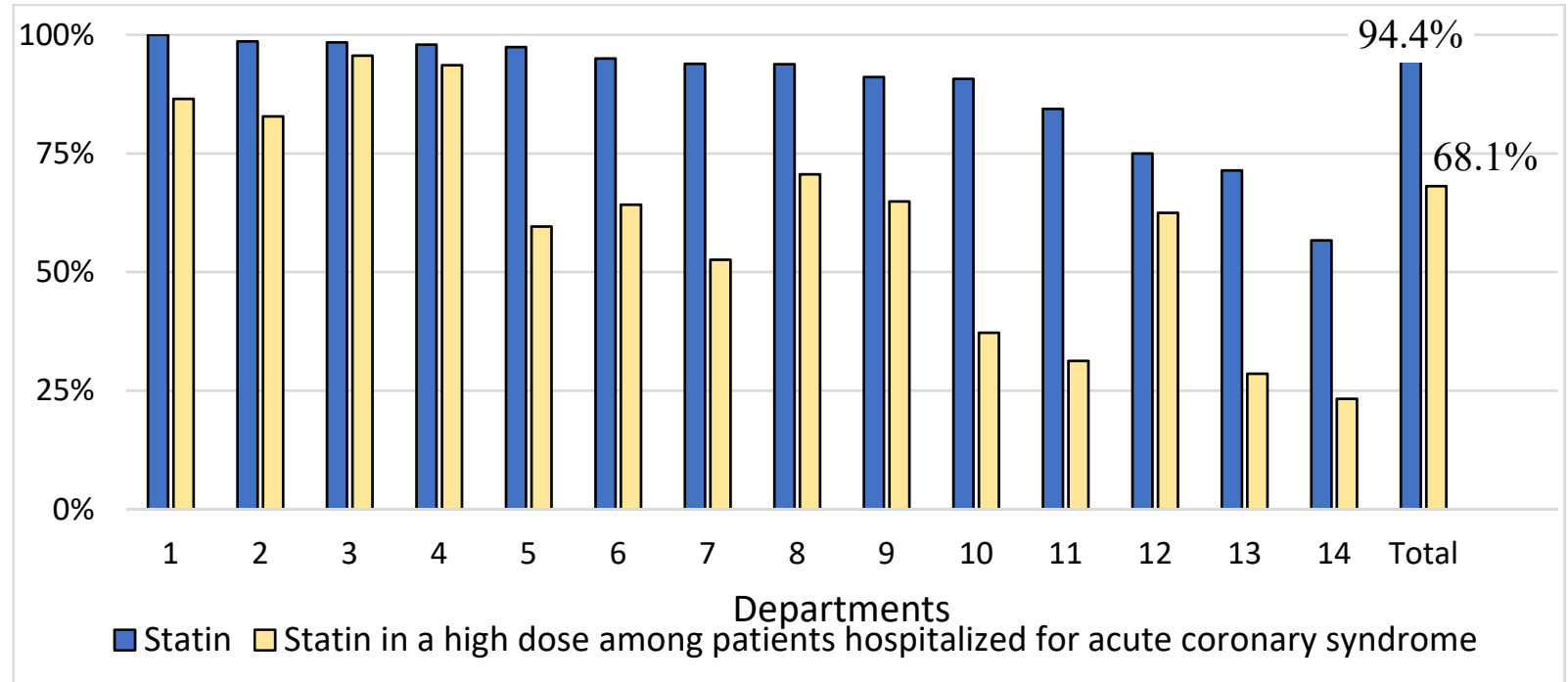

Figure 1. Proportions of patients prescribed a statin at discharge among all patients and statin in a high dose among those hospitalized for an acute coronary syndrome by departments.

Among patients hospitalized for ACS, a statin at any dose was prescribed to 683 (92.4\%) patients, whereas a high-dose statin was prescribed to $503(68.1 \%)$ patients with a significant variation between departments $(p<0.001$; Figure 1$)$. Hospitalization for myocardial infarction, hospitalization in a teaching hospital, cholesterol measurement during hospitalization and sex were independently associated with the prescription of statins (Table 2). Among patients prescribed a statin, 549 (80.6\%) were prescribed atorvastatin, 
$109(16.0 \%)$ were prescribed rosuvastatin, and $23(3.4 \%)$ were prescribed simvastatin. The mean doses of the drugs were atorvastatin at $48.2 \pm 27.0 \mathrm{mg}$ per day, rosuvastatin at $19.5 \pm 11.3 \mathrm{mg}$ per day, and simvastatin at $23.0 \pm 8.2 \mathrm{mg}$ per day. Among ACS patients, $19(2.6 \%)$ were prescribed a fibrate and $10(1.4 \%)$ were prescribed ezetimibe. Overall, 686 $(92.8 \%)$ of the ACS patients were prescribed at least one lipid-lowering drug.

\subsection{Management Following Discharge}

Overall, 1034 patients (83.7\%) attended the follow-up visit (12.0 \pm 3.7 months after their discharge from the hospital). The patients who attended did not differ significantly from those who did not participate in the follow-up examination with respect to age ( $64.6 \pm 8.5$ years vs. $65.7 \pm 8.3$ years; $p=0.08)$, sex (males: $71.6 \%$ vs. $70.0 \% ; p=0.63)$, index event $(p=0.12)$, and proportion of patients hospitalized in a teaching hospital (0.36). In nine cases, the blood cholesterol level was not measured. Therefore, we analyzed data of 1025 study participants in all subsequent analyses. A total of $918(89.6 \%)$ patients took a statin, and $803(78.3 \%)$ took a high-dose statin. Among patients prescribed a statin, $659(71.8 \%)$ were prescribed atorvastatin, $224(24.4 \%)$ rosuvastatin, and $35(3.8 \%)$ were prescribed simvastatin. The mean doses of statins were atorvastatin at $39.3 \pm 19.6 \mathrm{mg}$ per day, rosuvastatin at $20.4 \pm 11.1 \mathrm{mg}$ per day, and simvastatin at $23.1 \pm 9.9 \mathrm{mg}$ per day. Among those who did not change the statin they took between discharge and the follow-up examination, the mean dose of atorvastatin $(n=607)$ decreased by $7.0(4.9-9.2) \mathrm{mg}$ per day, whereas the mean doses of rosuvastatin and simvastatin did not change significantly. Among all the analyzed patients, $39(3.8 \%)$ patients were prescribed a fibrate and $27(2.6 \%)$ were prescribed ezetimibe. A statin plus a fibrate was used by $35(3.4 \%)$ patients, while a statin plus ezetimibe was taken by $23(2.2 \%)$ patients. Overall, $925(90.2 \%)$ patients took at least one lipid-lowering drug. Among patients prescribed a lipid-lowering drug at the time of hospital discharge, $896(91.4 \%)$ were taking a lipid-lowering drug at the time of the follow-up examination, whereas among those not prescribed any lipid-lowering drugs at the time of hospital discharge, 29 (64.4\%) used at least one cholesterol-lowering agent $(p<0.001)$. The intensity of lipid-lowering therapy from discharge to the follow-up interview increased in $177(17.3 \%)$ patients, decreased in $120(11.7 \%)$ patients, and remained unchanged in $728(71.0 \%)$ patients (Table 3$)$. Table 4 presents factors independently related to the use of lipid-lowering drugs at the time of the follow-up examination.

Table 3. Change in the lipid-lowering therapies from discharge to the follow-up examination.

\begin{tabular}{|c|c|c|}
\hline Prescribed at Discharge & Used at the Time of Interview & $n(\%)$ \\
\hline \multirow[t]{3}{*}{ No drugs $(n=45)$} & No drugs & $16(35.6)$ \\
\hline & Low/moderate-intensity therapy & $4(8.9)$ \\
\hline & High-intensity therapy & $25(55.6)$ \\
\hline \multirow{3}{*}{ Low / moderate-intensity therapy $(n=249)$} & No drugs & $29(11.8)$ \\
\hline & Low/moderate-intensity therapy & $72(28.9)$ \\
\hline & High-intensity therapy & $148(59.4)$ \\
\hline \multirow[t]{3}{*}{ High-intensity therapy $(n=731)$} & No drugs & $55(7.5)$ \\
\hline & Low/moderate-intensity therapy & $36(4.9)$ \\
\hline & High-intensity therapy & $640(87.6)$ \\
\hline
\end{tabular}


Table 4. Factors independently related to the use of at least one lipid-lowering drug and to achieving the recommended goal for LDL and non-HDL cholesterol in the post-discharge period.

Variable

Odds Ratio (95\% Confidence Intervals)

Factors Independently Related to the Use of at Least One Lipid-Lowering Drug in the Post-Discharge Period

Prescription of a lipid-lowering drug at discharge excluded from the statistical model

At least one consultation with a cardiologist in the post-discharge period

$2.45(1.50-4.00)$

Hospitalization in a teaching hospital

Professional activity

$1.97(1.22-3.17)$

Prescription of a lipid-lowering drug at discharge included in the statistical model

Prescription of a lipid-lowering drug at discharge

At least one consultation with a cardiologist in the post-discharge period

$5.88(3.05-11.34)$

$2.48(1.51-4.08)$

Factors Independently Related to Achieving the Recommended Goal for LDL and non-HDL Cholesterol in the Post-Discharge Period

Dependent variable: LDL cholesterol $<1.8 \mathrm{mmol} / \mathrm{L}$

Loneliness

Age, per 10 years

Employment

Diabetes

Statin prescribed at discharge

Dependent variable: non-HDL cholesterol $<2.6 \mathrm{mmol} / \mathrm{L}$

$\mathrm{CABG}$ as an index event

Smoking

Obesity

High blood pressure

Age, per 10 years

Males vs. females

High socio-economic status

Diabetes
$0.42(0.19-0.94)$
$1.32(1.10-1.59)$
$1.56(1.13-2.16)$
$1.66(1.27-2.16)$
$1.88(1.00-3.57)$

$0.52(0.27-0.98)$
$0.65(0.45-0.92)$
$0.73(0.56-0.94)$
$0.75(0.58-0.98)$
$1.31(1.10-1.54)$
$1.37(1.03-1.83)$
$1.41(1.06-1.88)$
$1.42(1.09-1.86)$

The mean level of total cholesterol was $4.12 \pm 1.12 \mathrm{mmol} / \mathrm{L}$, LDL cholesterol $2.19 \pm 0.95 \mathrm{mmol} / \mathrm{L}$, HDL cholesterol $1.32 \pm 0.38 \mathrm{mmol} / \mathrm{L}$, non-HDL cholesterol $2.80 \pm 1.08 \mathrm{mmol} / \mathrm{L}$, and triglycerides $1.50 \pm 0.99 \mathrm{mmol} / \mathrm{L}$ (median $1.29 \mathrm{mmol} / \mathrm{L}$, interquartile range $0.95-1.77 \mathrm{mmol} / \mathrm{L})$. Mean LDL cholesterol was $2.07 \pm 0.86 \mathrm{mmol} / \mathrm{L}$ in patients taking atorvastatin, $2.10 \pm 0.88 \mathrm{mmol} / \mathrm{L}$ in patients using rosuvastatin, $2.32 \pm 0.92 \mathrm{mmol} / \mathrm{L}$ in patients using simvastatin, and $3.07 \pm 1.13 \mathrm{mmol} / \mathrm{L}$ in those not using any statin. LDL cholesterol $<1.4 \mathrm{mmol} / \mathrm{L}$ was found in $162(15.8 \%)$ patients, $<1.8 \mathrm{mmol} / \mathrm{L}$ in $382(37.3 \%)$ patients, $<2.6 \mathrm{mmol} / \mathrm{L}$ in $761(74.2 \%)$, and $<4.0 \mathrm{mmol} / \mathrm{L}$ in 967 (94.3\%) patients, whereas non-HDL cholesterol $<2.2 \mathrm{mmol} / \mathrm{L},<2.6 \mathrm{mmol} / \mathrm{L},<3.4 \mathrm{mmol} / \mathrm{L}$, and $<4.8 \mathrm{mmol} / \mathrm{L}$ was observed in 303 (29.3\%), 519 (50.2\%), 806 (77.9\%), and 962 (93.0\%) participants, respectively. The factors independently related to achievement of the recommended goals are presented in Table 4.

\section{Discussion}

The key finding of this study is that despite overwhelming evidence that lipidlowering therapy decreases the risk of cardiovascular events in patients with coronary artery disease, $62.7 \%$ of study participants had LDL cholesterol levels above the recommended goal according to the 2016 guidelines [4]. Most of these patients probably failed to receive a proper evaluation and treatment, both in terms of pharmacotherapy as well as lifestyle modification, for dyslipidemia following hospitalization due to CAD. Overall, our results showed a sizeable potential for a further reduction in cardiovascular risk in patients with CAD through an improvement in hypercholesterolemia management. There is strong scientific evidence that the long-term survival of patients with CAD may be improved by providing optimal secondary prevention, including lipid-lowering treatment [4]. An 
even lower proportion of patients achieved the LDL cholesterol goal according to the most recent European Society of Cardiology guidelines [5].

Our results do not confirm the previous reports suggesting that the majority of patients discontinue their statin therapy within one year after initiation [26]. The intensity of lipidlowering therapy declined in the small portion of patients during the post-discharge period. However, it was increased only in $17 \%$ of study participants, although most patients had high LDL cholesterol levels. Combination therapy was rarely applied. In particular, ezetimibe and proprotein convertase subtilisin/kexin type 9 inhibitors were underutilized $[4,5,27,28]$. Indeed, one critically important issue of the lack of efficacy of the lipid-lowering therapy is the very low application of combination therapy. Considering the new recommendations, immediate application of the combination therapy with statin and ezetimibe, and even triple therapy in selected cases, especially for the very-high-risk patients, might significantly improve effectiveness of the lipid-lowering treatment $[5,29,30]$.

Hospitalization in a teaching hospital almost doubled the likelihood of a patient taking a lipid-lowering drug following discharge according to the report that analyzed data of patients hospitalized for CAD between 1996 and 1999 [31]. Our results suggest this association has not changed since the end of 20th century, despite multiple factors including several educational initiatives organized for physicians and profound changes in European societies induced by the collapse of the communist system in 1989 and the enlargement of the European Union. The Polish healthcare system has been in change for the last 20 years, both in terms of institutional changes and regulations regarding drug registration, prescription, and reimbursement. Importantly, the relative position of primary care physicians and specialists has evolved. In this respect, it is worth noting that the relationship between lipid-lowering drug use and practice settings has not changed significantly [31]. We showed a considerable variation in the use of lipid-lowering therapies among cardiac departments in addition to the previously found differences among countries [6]. The evidence suggests the variation has not decreased significantly over the last 20 years $[32,33]$.

Our results suggest that specialized cardiology care is independently related to higher lipid-lowering drug utilization. There are several possible explanations for this finding. Firstly, patients consulted and not consulted by a cardiologist may differ with respect to a number of unrecognized factors, including greater compliance with the physicians' recommendations. Secondly, cardiologists may dedicate more time to controlling cardiovascular risk factors compared to family doctors. Finally, a specialist usually has a greater authority than a family doctor and this could also partially explain the finding. The relationship between a consultation with a cardiologist and the proportion of patients with a cholesterol level at the recommended goal could help explain the lower risk of death among post-infarction patients who consulted a cardiologist [34].

Among patients analyzed in the Euroaspire V survey, no correlation was observed between a patient's level of education and the LDL cholesterol level following hospitalization due to CAD [20]. Our results are concordant with the analysis conducted by De Bacquer et al., as there was no observed correlation between an individual's level of education and ensuring the appropriate control of cholesterol level in our study group. We showed that a high socio-economic status and loneliness are independently related to the effectiveness of hypercholesterolemia management in patients with coronary artery disease. These results expand on earlier findings of the relationship between loneliness and risk factor control [20].

A lack of employment was independently associated with a high LDL cholesterol level. This might be due to financial barriers, but other factors (e.g., number of comorbidities) may also be responsible for this finding. In addition, control of hypercholesterolemia was related to smoking, obesity, and high blood pressure. This may underline the importance of inappropriate lifestyle modification and low adherence to prescribed therapies. Importantly, participation in a cardiac rehabilitation program was not independently related to the probability of LDL cholesterol levels at the recommended goal. 
Finally, we found females to be prescribed a statin at discharge significantly less frequently. Females were also less likely to achieve the recommended non-HDL cholesterol level in the post-discharge period. The present results support the findings from the multicenter survey EUROASPIRE V [35]. This finding suggests the sex-gap in risk factor control of coronary patients is far from closing in many European countries. Further actions are needed to increase the awareness of the worse cholesterol control in female patients.

\section{Limitations}

Besides the design of the study which precluded any consideration of causality, the present analysis has several other limitations. Firstly, we could not assess the association between hypercholesterolemia management and the risk of cardiovascular events. Secondly, our study participants were not representative of all coronary artery disease patients. The analyzed patients were limited to those who had experienced an acute CAD event or had undergone a myocardial revascularization procedure. Thirdly, we did not include patients aged over 80 years. Fourthly, we could not assess adherence to medications. The assessment of adherence would have increased the impact of our study. Conversely, an important advantage of our analysis is that our results are not based only on abstracted medical record data only, but involved face-to-face interviews and examinations using the same protocol and standardized methods and instruments. Thus, to the best of our knowledge, the current findings provide the most trustworthy data on cholesterol management for secondary prevention of CAD.

\section{Conclusions}

Health-system-related factors are associated with the utilization of lipid-lowering drugs, whereas mainly patient-related factors are related to the control of hypercholesterolemia following hospitalization due to coronary artery disease. Our results may help in the task of developing strategies to improve hypercholesterolemia management in patients with CAD.

Author Contributions: K.K. (Kornelia Kotseva), D.W., D.D.B., G.D.B., P.J. and A.P. contributed to the design of the research. P.J. conceived the idea for the analysis. P.J., P.K., M.S., M.P., M.H., M.B., Z.G., K.K. (Karol Kamiński) and D.A.K. were involved in data collection. P.J. analyzed the data and drafted the manuscript. All authors have edited, read and agreed to the published version of the manuscript.

Funding: The project was supported by the Jagiellonian University (K/ZDS/006429) and the Medical University of Białystok (SUB/1/DN/20/001/1201) grants.

Institutional Review Board Statement: The study was conducted according to the guidelines of the Declaration of Helsinki, and approved by the institutional Bioethics Committees.

Informed Consent Statement: Informed consent was obtained from all subjects involved in the study.

Data Availability Statement: Data available on request only for scientific purposes.

Acknowledgments: The authors are grateful to the administrative staff, physicians, nurses, and other personnel in the hospitals in which the survey was carried out and to all patients who participated in the surveys.

Conflicts of Interest: P.J. has received honoraria, research and travel grants from Amgen, KRKA, Novartis, SanofiAventis, Servier.

\section{References}

1. On behalf of the Atlas Writing Group; Timmis, A.; Townsend, N.; Gale, C.P.; Torbica, A.; Lettino, M.; E Petersen, S.; A Mossialos, E.; Maggioni, A.P.; Kazakiewicz, D.; et al. European Society of Cardiology: Cardiovascular Disease Statistics 2019 (Executive Summary). Eur. Hear. J. Qual. Care Clin. Outcomes 2020, 6, 7-9. [CrossRef]

2. Dorresteijn, J.A.; Visseren, F.J.; Wassink, A.M.; Gondrie, M.J.; Steyerberg, E.W.; Ridker, P.M.; Cook, N.R.; van der Graaf, Y.; on behalf of the SMART Study Group. Development and validation of a prediction rule for recurrent vascular events based on a cohort study of patients with arterial disease: The SMART risk score. Heart 2013, 99, 866-872. [CrossRef] 
3. Koskinas, K.C.; Siontis, G.C.; Piccolo, R.; Mavridis, D.; Räber, L.; Mach, F.; Windecker, S. Effect of statins and non-statin LDLlowering medications on cardiovascular outcomes in secondary prevention: A meta-analysis of randomized trials. Eur. Heart $J$. 2018, 39, 1172-1180. [CrossRef]

4. $\quad$ Piepoli, M.F.; Hoes, A.W.; Agewall, S.; Albus, C.; Brotons, C.; Catapano, A.L.; Cooney, M.T.; Corrà, U.; Cosyns, B.; Deaton, C.; et al. The Sixth Joint Task Force of the European Society of Cardiology and Other Societies on Cardiovascular Disease Prevention in Clinical Practice. Eur. Heart J. 2016, 37, 2315-2381. [CrossRef]

5. Mach, F.; Baigent, C.; Catapano, A.L.; Koskinas, K.C.; Casula, M.; Badimon, L.; Chapman, M.J.; De Backer, G.G.; Delgado, V.; Ference, B.A.; et al. 2019 ESC/EAS Guidelines for the management of dyslipidaemias: Lipid modification to reduce cardiovascular risk: The Task Force for the management of dyslipidaemias of the European Society of Cardiology (ESC) and European Atherosclerosis Society (EAS). Eur. Heart J. 2020, 41, 111-188. [CrossRef]

6. De Backer, G.; Jankowski, P.; Kotseva, K.; Mirrakhimov, E.; Reiner, Ž.; Rydén, L.; Tokgözoğlu, L.; Wood, D.; De Bacquer, D.; EUROASPIRE V Collaborators. Management of dyslipidaemia in patients with coronary heart disease: Results from the ESC-EORP EUROASPIRE V survey in 27 countries. Atherosclerosis 2019, 285, 135-146. [CrossRef]

7. Ferrieres, J.; De Ferrari, G.M.; Hermans, M.P.; Elisaf, M.; Toth, P.P.; Horack, M.; Brudi, B.; Lautsch, D.; Bash, L.D.; Baxter, C.A.; et al. Predictors of LDL-cholesterol target value attainment differ in acute and chronic coronary heart disease patients: Results from DYSIS II Europe. Eur. J. Prev. Cardiol. 2018, 25, 1966-1976. [CrossRef] [PubMed]

8. Jankowski, P.; Kosior, D.A.; Sowa, P.; Szóstak-Janiak, K.; Kozieł, P.; Krzykwa, A.; Sawicka, E.; Haberka, M.; Setny, M.; Kamiński, K.; et al. Secondary prevention of coronary artery disease in Poland. Results from the POLASPIRE survey. Cardiol. J. 2020, 27, 533-540.

9. Ray, K.K.; Molemans, B.; Schoonen, W.M.; Giovas, P.; Bray, S.; Kiru, G.; Murphy, J.; Banach, B.; De Servi, S.; Gaita, D.; et al. EU-wide cross-sectional observational study of lipid-modifying therapy use in secondary and primary care: The DA VINCI study. Eur. J. Prev. Cardiol. 2021, in press.

10. Rosenson, R.S.; Kent, S.T.; Brown, T.M.; Farkouh, M.E.; Levitan, E.B.; Yun, H.; Sharma, P.; Safford, M.M.; Kilgore, M.; Muntner, P.; et al. Underutilization of high-intensity statin therapy after hospitalization for coronary heart disease. J. Am. Coll. Cardiol. 2015, 65, 270-277. [CrossRef] [PubMed]

11. Kotseva, K.; De Backer, G.; De Bacquer, D.; Rydén, L.; Hoes, A.; Grobbee, D.; Maggioni, A.; Marques-Vidal, P.; Jennings, C.; Abreu, A.; et al. Lifestyle and impact on cardiovascular risk factor control in coronary patients across 27 countries: Results from the European Society of Cardiology ESC-EORP EUROASPIRE V registry. Eur. J. Prev. Cardiol. 2019, 26, 824-835. [CrossRef] [PubMed]

12. Frank-Tewaag, J.; Bleek, J.; Horenkamp-Sonntag, D.; Marschall, U.; Zeymer, U.; Donner-Banzhoff, N.; Sundmacher, L. Use of guideline-recommended drug therapy in patients undergoing percutaneous coronary intervention for stable coronary heart disease in Germany: A multilevel analysis of nationwide routine data. BMJ Open 2020, 10, e042886. [CrossRef]

13. Roth, G.A.; Mensah, G.A.; Johnson, C.O.; Addolorato, G.; Ammirati, E.; Baddour, L.M.; Barengo, N.C.; Beaton, A.Z.; Benjamin, E.J.; Benziger, C.P.; et al. Global Burden of Cardiovascular Diseases and Risk Factors, 1990-2019: Update from the GBD 2019 Study. J. Am. Coll. Cardiol. 2020, 76, 2982-3021. [CrossRef]

14. Harris, M.F.; Parker, S.M.; Litt, J.; Van Driel, M.; Russell, G.; Mazza, D.; Jayasinghe, U.W.; Del Mar, C.; Lloyd, J.; Smith, J.; et al. Implementing guidelines to routinely prevent chronic vascular disease in primary care: The Preventive Evidence into Practice cluster randomised controlled trial. BMJ Open 2015, 5, e009397. [CrossRef]

15. Devi, R.; Singh, S.J.; Powell, J.; Fulton, E.; Igbinedion, E.; Rees, K. Internet-based interventions for the secondary prevention of coronary heart disease. Cochrane Database Syst. Rev. 2015, 12, CD009386. [CrossRef] [PubMed]

16. Jakobsson, S.; Irewall, A.-L.; Bjorklund, F.; Mooe, T. Cardiovascular secondary prevention in high-risk patients: A randomized controlled trial sub-study. BMC Cardiovasc. Disord. 2015, 15, 1-8. [CrossRef]

17. Pająk, A.; Wolfshaut-Wolak, R.; Doryńska, A.; Jankowski, P.; Fornal, M.; Grodzicki, T.; Jennings, C.; Kawecka-Jaszcz, K.; Kotseva, K.; Pajak, K.; et al. Longitudinal effects of a nurse-managed comprehensive cardiovascular disease prevention program for hospitalized coronary heart disease patients and primary care high-risk patients. Kardiologia Polska 2020, 78, 429-437. [CrossRef] [PubMed]

18. Bustillo, S.R.; Ivern, C.; Badosa, N.; Farre, N.; Marco, E.; Bruguera, J.; Cladellas, M.; Enjuanes, C.; Cainzos-Achirica, M.; Marti-Almor, J.; et al. Efficacy of a nurse-led lipid-lowering secondary prevention intervention in patients hospitalized for ischemic heart disease: A pilot randomized controlled trial. Eur. J. Cardiovasc. Nurs. 2019, 18, 366-374. [CrossRef]

19. Jankowski, P.; Niewada, M.; Bochenek, A.; Bochenek-Klimczyk, K.; Bogucki, M.; Drygas, W.; Dudek, D.; Eysymontt, Z.; Grajek, S.; Kozierkiewicz, A.; et al. Optimal Model of Comprehensive Rehabilitation and Secondary Prevention. Kardiologia Polska 2013, 71, 995-1003. [CrossRef]

20. De Bacquer, D.; van de Luitgaarden, I.A.; De Smedt, D.; Vynckier, P.; Bruthans, J.; Fras, Z.; Jankowski, P.; Dolzhenko, M.; Kotseva, K.; Wood, D.; et al. Socioeconomic characteristics of patients with coronary heart disease in relation to their cardiovascular risk profile. Heart 2021, 107, 799-806. [CrossRef] [PubMed]

21. Kubica, A.; Kasprzak, M.; Obonska, K.; Fabiszak, T.; Laskowska, E.; Navarese, E.; Kozinski, M.; Sztuba, B.; Swiatkiewicz, I.; Grzesk, G.; et al. Discrepancies in Assessment of Adherence to Antiplatelet Treatment after Myocardial Infarction. Pharmacology 2015, 95, 50-58. [CrossRef] 
22. Jankowski, P.; Czarnecka, D.; Łukaszewska, A.; Łysek, R.; Wolfshaut-Wolak, R.; Bogacki, P.; Grodecki, J.; Mirek-Bryniarska, E.; Nessler, J.; Podolec, P.; et al. Factors related to the effectiveness of hypercholesterolemia treatment following hospitalization for coronary artery disease. Pol. Arch. Intern. Med. 2016, 126, 388-394. [CrossRef]

23. Kozieł, P.; Jankowski, P.; Kosior, D.A.; Sowa, P.; Szóstak-Janiak, K.; Krzykwa, A.; Sawicka, E.; Haberka, M.; Setny, M.; Kamiński, K.; et al. Smoking cessation in patients with established coronary artery disease: Data from the POLASPIRE survey. Kardiologia Polska 2021, 79, 418-425. [CrossRef]

24. Zigmond, A.S.; Snaith, R.P. The hospital anxiety and depression scale. Acta Psych. Scand. 1983, 67, 361-370. [CrossRef]

25. Levey, A.S.; Coresh, J.; Greene, T.; Stevens, L.A.; Zhang, Y.; Hendriksen, S.; Kusek, J.W.; Van Lente, F. Using standardized serum creatinine values in the modification of diet in renal disease study equation for estimating glomerular filtration rate. Ann. Intern. Med. 2006, 145, 247-254. [CrossRef]

26. Banach, M.; Štulc, T.; Dent, R.; Toth, P.P. Statin non-adherence and residual cardiovascular risk: There is need for substantial improvement. Int. J. Cardiol. 2016, 225, 184-196. [CrossRef] [PubMed]

27. Banach, M.; Jankowski, P.; Jóźwiak, J.; Cybulska, B.; Windak, A.; Guzik, T.; Mamcarz, A.; Broncel, M.; Tomasik, T. PoLA/CFPiP/PCS Guidelines for the Management of Dyslipidaemias for Family Physicians 2016. Arch. Med. Sci. 2017, 1, 1-45. [CrossRef]

28. Harris, E.D.; Lacey, A.; Akbari, A.; Torabi, F.; Smith, D.; Jenkins, G.; Obaid, D.; Chase, A.; Gravenor, M.; Halcox, J. Achievement of European guideline-recommended lipid levels post-percutaneous coronary intervention: A population-level observational cohort study. Eur. J. Prev. Cardiol. 2021, 28, 854-861. [CrossRef]

29. Averna, M.; Banach, M.; Bruckert, E.; Drexel, H.; Farnier, M.; Gaita, D.; Magni, P.; März, W.; Masana, L.; e Silva, A.M.; et al. Practical guidance for combination lipid-modifying therapy in high- and very-high-risk patients: A statement from a European Atherosclerosis Society Task Force. Atherosclerosis 2021, 325, 99-109. [CrossRef]

30. Banach, M.; Penson, P.E. Lipid-lowering therapies: Better together. Atherosclerosis 2021, 320, 86-88. [CrossRef]

31. Kawecka-Jaszcz, K.; Jankowski, P.; Pajak, A. Determinants of appropriate lipid management in patients with ischaemic heart disease. Cracovian Program for Secondary Prevention of Ischaemic Heart Disease. Int. J. Cardiol. 2003, 91, 15-23. [CrossRef]

32. EUROASPIRE II Study Group. Lifestyle and risk factor management and use of drug therapies in coronary patients from 15 countries; principal results from EUROASPIRE II Euro Heart Survey Programme. Eur. Heart J. 2001, 22, 554-572. [CrossRef] [PubMed]

33. Kawecka-Jaszcz, K.; Jankowski, P.; Pająk, A.; Dubiel, J.S.; Maciejewicz, J.; Piotrowski, W.; Śmielak-Korombel, W.; Tracz, W. Cracovian program for secondary prevention of ischaemic heart disease. Secondary prevention of ischaemic heart disease during hospitalisation in 1996/1997 and 1998/1999. Eur Heart J. Suppl. 2004, 6 (Suppl. J), J47-J52.

34. Pęksa, J.W.; Storman, D.; Jankowski, P.; Staśkiewicz, W.; Jasińska, K.W.; Czarnecka, D.; Bała, M.M. Mortality in patients after acute myocardial infarction managed by cardiologists and primary care physicians: A systematic review. Pol. Arch. Intern. Med. 2020, 130, 860-867. [CrossRef]

35. Vynckier, P.; Ferrannini, G.; Rydén, L.; Jankowski, P.; De Backer, T.; Gevaert, S.; De Bacquer, D.; De Smedt, D. On behalf of the EUROASPIRE V Investigators group Gender gap in risk factor control of coronary patients far from closing: Results from the European Society of Cardiology EUROASPIRE V registry. Eur. J. Prev. Cardiol. 2020. [CrossRef] [PubMed] 